\title{
Prevalência e fatores sociodemográficos associados à fragilidade em mulheres idosas
}

\author{
Prevalence and sociodemographic factors associated with frailty in elderly women \\ Prevalencia y factores sociodemográficos asociados a la fragilidad en mujeres ancianas
}

\section{Marcella Costa Souto Duarte', Maria das Graças Melo Fernandes", Rosalina Aparecida Partezani Rodrigues"', Maria Miriam Lima da Nóbrega"}

\author{
'Centro Universitário de João Pessoa - UNIPÊ, Curso de Graduação em Enfermagem. João Pessoa-PB, Brasil. \\ "Universidade Federal da Paraíba, Centro de Ciências da Saúde, \\ Programa de Pós-Graduação em Enfermagem. João Pessoa-PB, Brasil. \\ I' Universidade de São Paulo, Escola de Enfermagem de Ribeirão Preto, \\ Departamento de Enfermagem Geral e Especializada. Ribeirão Preto-SP, Brasil.
}

Submissão: 02-06-2012 Aprovação: 20-11-2013

\section{RESUMO}

Estudo transversal, que objetivou de estimar a prevalência de fragilidade em mulheres idosas, residentes no município de João Pessoa, Paraíba, Brasil; e identificar possíveis associações entre a fragilidade e as variáveis sociodemográficas. A amostra foi composta por 166 idosas, entrevistadas nos domicílios, entre abril e junho de 2011. Para a coleta dos dados, utilizou-se instrumento estruturado com questões relativas às variáveis sociodemográficas e à Edmonton Frail Scale. A análise descritiva dos dados, realizada pelo programa SPSS 15.0, mostrou que a maioria das idosas $(60,8 \%)$ evidenciava algum grau de fragilidade. Entre elas, $21,7 \%$ eram aparentemente vulneráveis, $23,5 \%$, com fragilidade leve, $7,8 \%$, moderada, e 7,8\%, e grave. Verificou-se associação do fenômeno com idade, escolaridade e renda, condições sobre as quais os enfermeiros devem atuar com vistas à prevenção do evento.

Descritores: Mulher; Idoso Fragilizado; Envelhecimento; Enfermagem.

\section{ABSTRACT}

Cross-sectional study that aimed to estimating the prevalence of frailty in older women living in the city of João Pessoa, Paraíba, Brazil; and to identify possible associations between frailty and socio-demographic variables. The sample included 166 elderly women were interviewed in their home between April and June 2011. For data was collected using a structure instrument to questions on socio-demographic variables and the Edmonton Frail Scale. The descriptive data analysis, performed by SPSS 15.0, showed that most elderly women $60.8 \%$ showed some degree of fragility. Among them, $21.7 \%$ were apparently vulnerable, $23.5 \%$ mild frailty, $7.8 \%$ moderate and $7.8 \%$ severe frailty. It was found an association of the phenomena with age, education and income, conditions under which nurses must act in order to prevent the event.

Key words: Women; Frail Elderly; Aging; Nursing.

\section{RESUMEN}

Estudio transversal que objetivó estimar la prevalencia de fragilidad en mujeres adultas mayores residentes en el municipio de João Pessoa, Paraíba, Brasil; y identificar posibles asociaciones entre fragilidad y variables socio-demográficas. La muestra comprendió 166 mujeres ancianas, entrevistadas en los domicilios entre abril a junio de 2011. Para la colecta de datos se utilizo un instrumento estructurado con preguntas relativas a las variables socio-demográficas y la Edmonton Frail Scale. El análisis descriptiva de los datos, realizado por el programa SPSS 15.0, mostro que la mayoría de las adultas mayores $(60.8 \%)$ evidenciaban algún grado de fragilidad. Entre ellas, $21.7 \%$ eran aparentemente vulnerables, $23.5 \%$ evidenciaban fragilidad leve, $7.8 \%$ fragilidad moderada y $7.8 \%$ fragilidad grave. Se verifico asociación del fenómeno con la edad, escolaridad e ingresos mensuales, condiciones sobre las cuales los enfermeros deben actuar con vistas a la prevención del evento.

Palabras clave: Mujer; Adulto Mayor Fragilizado; Envejecimiento; Enfermería. 


\section{INTRODUÇÃO}

O crescimento da população idosa tem sido tema constante de debate entre pesquisadores, gestores sociais e profissionais da saúde, pois se trata de uma realidade mundial, que determina importantes repercussões nos âmbitos social e no econômico, devido a um declínio das taxas de natalidade e de mortalidade, resultante de alterações no perfil da morbidade, consequente à melhoria da qualidade de vida e aos avanços da área de saúde ${ }^{(1)}$

No âmbito nacional, há 20,5 milhões de idosos, cerca de $10 \%$ da população do país ${ }^{(2)}$. Estimativas do Ministério da Saúde mostram que, em 2050, o número de idosos deverá chegar a 56 milhões, o que representará $24 \%$ da população ${ }^{(2)}$ e um incremento do número de mulheres idosas - evento que já constitui uma realidade atual, categorizado como a "feminização da veIhice". Esse fenômeno é um processo de transição relacionada ao gênero. $\mathrm{O}$ aspecto central da transição de gênero diz respeito às mudanças nos padrões de sobrevivência de homens e de muIheres, o que determina diferenças nas formas de adoecer e de como se cuidar, verificadas entre eles, além de sua capacidade para o desempenho de funções ou sua vulnerabilidade ${ }^{(3)}$.

Com o aumento da proporção de idosos, de sua expectativa de vida, emergem as doenças crônicas não transmissíveis e consequentemente as incapacidades. O comprometimento do idoso por causa dessas enfermidades traz desfechos negativos para ele, sua família, comunidade e o sistema de saúde, uma vez que ocasiona mais vulnerabilidade e dependência, causadas pelas perdas funcionais em seus diferentes sistemas orgânicos, o que culmina, em geral, em um quadro de fragilidade ${ }^{(4)}$, condição caracterizada pela diminuição das reservas fisiológicas, que está associada a um aumento do risco de incapacidade funcional e mais vulnerabilidade à morbidade e à mortalidade ${ }^{(5)}$.

A despeito disso, os estudos acerca da incidência, da prevalência e dos fatores associados à fragilidade em idosos ${ }^{(6)}$ ainda são escassos, razão por que é preciso avaliar tais aspectos nesse grupo populacional, especialmente aqueles que vivem na comunidade, com destaque para as mulheres. Esses achados poderão contribuir para o preenchimento de lacunas relativas à inexistência ou à insuficiência de dados empíricos sobre o fenômeno e para a melhoria da assistência em saúde prestada aos idosos, particularmente, para o cuidado de enfermagem.

Considerando os fatores associados à fragilidade entre os idosos, diferentes estudos têm apontado fatores sociodemográficos por afetar, especialmente, seu estilo de vida e aumentar sua exposição a riscos para a saúde ${ }^{(6-7)}$. Assim, visando identificar tal realidade, com base em um estudo de base populacional, no cenário local, foram delimitados para este estudo os seguintes objetivos: estimar a prevalência de fragilidade em mulheres idosas, residentes no município de João Pessoa, Paraíba, Brasil; e identificar possíveis associações entre a fragilidade e as variáveis sociodemográficas.

\section{REVISÃO DA LITERATURA}

No período anterior à década de 1980, os termos fragilidade e idoso frágil foram pouco descritos na literatura científica.
A partir da década de 1980, houve um aumento do número de estudos acerca da fragilidade e, nessa época, as definições encontradas para idoso frágil incluíam características como idade igual ou superior a 75 anos, vulnerabilidade, déficit físico e/ou cognitivo, participação em programa geriátrico, necessidade de cuidados institucionais e dependência para realizar as $\mathrm{AVDs}^{(7)}$. Tais definições estabeleceram relações de equivalência entre incapacidade e fragilidade, e a entidade passou a ser descrita por meio de expressões como comorbidade, institucionalização, idade avançada, condição pré-óbito e incapacidade decorrente de doenças crônicas. Essas definições, no entanto, foram consideradas inadequadas, por reforçar estereótipos negativos relacionados ao envelhecimento ${ }^{(8)}$.

Em 1990, no índice remissivo do Journal of the American Geriatrics Society, foi publicado, pela primeira vez, o termo idoso frágil, despertando o interesse de pesquisadores pela temáti$\mathrm{ca}^{(7)}$. Nesse mesmo período, a partir de estudos longitudinais, em que se mostrava a existência de idosos com incapacidades, mas considerados não frágeis e, por outro lado, idosos frágeis, que não apresentavam incapacidades, passou-se a questionar a equivalência entre os dois termos, o que levou o conceito ser frágil a ser, gradativamente, substituído por tornar-se frágil(9), pois se passou a acreditar que a fragilidade poderia estar desvinculada das doenças crônicas não-transmissíveis, da dependência funcional e da necessidade de receber assistência social.

Existem, atualmente, dois grupos de pesquisa internacionais que têm se destacado no trabalho, com vistas a alcançar uma definição consensual do conceito de fragilidade e estudos de instrumentos para sua operacionalização: nos Estados Unidos, na John Hopkins University; e no Canadá, o Canadian Iniciative on Frailty and Aging (CIF-A).

Na John Hopkins University, nos Estados Unidos, realizou-se estudo com fim de estabelecer critérios objetivos mensuráveis para definir a fragilidade em idosos com base na hipótese de que ela representa uma síndrome que pode ser identificada através de um fenótipo. Com base nessa proposição e em sua aplicação em um grupo de 5.317 idosos, com idade $\geq 65$ anos, que viviam na comunidade, os autores do referido estudo observaram a prevalência da síndrome de 6,9\% e incidência de $7,2 \%$, em quatro anos, com predominância entre as mulheres ${ }^{(5)}$.

A pesquisa Canadian Iniciative on Frailty and Aging (CIF-A), desenvolvida entre o Canadá, a União Europeia, Israel e o Japão, iniciada em 2002, busca ampliar o conhecimento sobre fragilidade em idosos, mediante estudo minucioso das causas e da trajetória do fenômeno, com ênfase na prevenção e no tratamento, considerando o fenômeno numa perspectiva multidimensional. Nesse pressuposto, a identificação da fragilidade no idoso envolve a avaliação de elementos como cognição, humor e suporte social ${ }^{(10)}$. Em consonância com essa perspectiva, na atualidade, compreende-se a fragilidade como um evento determinado por aspectos biológicos e psicossociais. Essa forma de entender o fenômeno foi adotada no âmbito deste estudo.

\section{MÉTODO}

Trata-se de uma pesquisa transversal, realizada na cidade de João Pessoa, capital do estado da Paraíba, Brasil. Constitui-se 
em um subprojeto da pesquisa "Condições de vida, saúde e envelhecimento: um estudo comparado", financiada pelo Programa Nacional de Cooperação Acadêmica (PROCAD/ CAPES) entre a Universidade Federal da Paraíba e a Escola de Enfermagem de Ribeirão Preto, da Universidade de São Paulo.

A população de base para o cálculo amostral compreendeu a população idosa, de 60 anos ou mais, do referido município. Participaram da amostra, cujo processo foi probabilístico, por conglomerados e de duplo estágio, 166 mulheres idosas. Os critérios empregados para a seleção das participantes foram: ter idade igual ou superior a 60 anos e residir nos setores censitários do município delimitados no processo de amostragem.

A coleta dos dados foi realizada por meio de entrevista no domicílio dos idosos, no período de abril a junho de 2011, por alunos de graduação e de pós-graduação. As variáveis mensuradas nesta pesquisa foram idade, renda, escolaridade e presença de fragilidade. Quanto aos instrumentos para a coleta das informações, foram empregados um roteiro estruturado contendo questões específicas para identificar as variáveis sociodemográficas e a Edmonton Frail Scale - EFS, que identifica a fragilidade a partir de nove domínios: cognição, estado geral de saúde, independência funcional, suporte social, uso de medicamentos, nutrição, humor, continência e desempenho funcional. Essa escala foi desenvolvida e validada por participantes da pesquisa Canadian Iniciative on Frailty and Aging (CIF-A) $)^{(11)}$, que a consideram um instrumento mais completo, confiável e de fácil manuseio para a detecção de fragilidade em pessoas idosas. No Brasil, a EFS foi validada e adaptada culturalmente numa população de idosos do município de Ribeirão Preto, São Paulo(12).

No que concerne à análise dos dados, inicialmente, eles foram armazenados em uma planilha eletrônica estruturada no Microsoft Excel 2010 for Windows. Foi feita dupla digitação, no sentido de promover a eliminação de erros e garantir a confiabilidade na compilação dos dados. Em seguida, as informações foram exportadas para o programa Statistical Package for the Social Sciences - SPSS, versão 15.0, para a efetivação de análise quantitativa das variáveis, por meio de estatística descritiva. A análise descritiva foi desenvolvida utilizando-se média, desvio-padrão e frequências absoluta e relativa. Na etapa da análise confirmatória, foram construídas tabelas de contingência para as variáveis qualitativas e aplicado o teste Qui-Quadrado de Pearson $\left(\mathrm{x}^{2}\right)$ ou teste Exato de Fisher para identificar possível associação entre as variáveis independentes (sociodemográficas) e a variável dependente (fragilidade). Para as variáveis quantitativas, foi utilizada a Análise de Variância (ANOVA) ou o teste de Mann-Whitney, e, para verificar a correlação entre essas variáveis, empregou-se o teste de Pearson ou o teste não paramétrico de Spearman. Foi considerado um nível de significância de $5 \%$.

Quanto aos aspectos éticos, a pesquisa foi realizada após aprovação do Comitê de Ética em Pesquisa do Hospital Universitário Lauro Wanderley, da Universidade Federal da Paraíba, por meio do protocolo de $n^{\circ} .680 / 10$. Todas as participantes receberam e assinaram o Termo de Consentimento Livre e Esclarecido (TCLE), conforme recomenda a Resolução n. 196/96 do Conselho Nacional de Saúde ${ }^{(13)}$.

\section{RESULTADOS}

\section{Caracterização sociodemográfica das participantes do estudo}

Das 166 mulheres idosas que participaram do estudo, 41 $(24,7 \%)$ se encontram na faixa etária de 80 anos ou mais. A idade variou entre 60 e 96 anos, com média de 73,25. Também se observou predominância de mulheres idosas de pele branca, 70 (42,2\%). Em relação ao estado civil, 70 mulheres são viúvas $(42,2 \%)$. No que diz respeito à assistência à saúde, 100 (60,2\%) idosas utilizavam o Sistema Único de Saúde (SUS).

Quanto à moradia, constatou-se que 71 mulheres (43,3\%) residiam no mesmo endereço há trinta anos ou mais e que 40 $(24,1 \%)$ moravam com quatro pessoas no domicílio. No tocante às características do arranjo familiar, observou-se que 50 $(30,1 \%)$ eram de natureza trigeracional (idoso, filhos e netos) e chefiado pelas próprias idosas (48,8\%). Esse arranjo passou a ter essa composição, em especial, devido ao fato de seus familiares $(36,7 \%)$ irem morar com a idosa. No concernente ao número de filhos, 59 (35,5\%) mulheres tinham de três a cinco filhos.

Em relação ao nível de escolaridade das idosas, a média de anos de estudo equivale a 6,48 e DP de 6,1. Entre elas, 48 $(28,9 \%)$ têm entre cinco e oito anos de estudo, e $45(27,1 \%)$ são analfabetas. Quanto à renda individual, a média foi de R\$ $1.299,38$, e a média da renda familiar, incluindo a da idosa, foi de $\mathrm{R} \$ 2.990,23$. Sobre o tipo de renda, a 123 (74,1\%) idosas receberam aposentadoria (Tabela 1 ).

Tabela 1 - Distribuição das idosas, segundo escolaridade e renda, João Pessoa - PB, Brasil, 2011 ( $n=166)$.

\begin{tabular}{|c|c|c|}
\hline Variáveis & Número & $\%$ \\
\hline \multirow[t]{2}{*}{ Idade } & Média (DP) & $73,25(8,91)$ \\
\hline & Mediana & 73,0 \\
\hline $60-64$ & 34 & $20,5 \%$ \\
\hline $65-69$ & 29 & $17,5 \%$ \\
\hline $70-74$ & 34 & $20,5 \%$ \\
\hline $75-79$ & 28 & $16,9 \%$ \\
\hline $80-85$ & 22 & $13,3 \%$ \\
\hline $86-89$ & 12 & $7,2 \%$ \\
\hline 90 anos ou mais & 7 & $4,2 \%$ \\
\hline Quantos anos frequentou a escola & Média (DP) & $6,48(6,10)$ \\
\hline \multicolumn{3}{|l|}{ Escolaridade (anos de estudo) } \\
\hline Analfabeto & 45 & $27,1 \%$ \\
\hline $0-4$ anos & 25 & $15,1 \%$ \\
\hline 5-8 anos & 48 & $28,9 \%$ \\
\hline 9-11 anos & 15 & $9,0 \%$ \\
\hline 12 ou mais anos & 33 & $19,9 \%$ \\
\hline \multicolumn{3}{|l|}{ Renda Mensal } \\
\hline Idosa & Média & $\mathrm{R} \$ 1.299,38$ \\
\hline Até 1 salário mínimo & 13 & $7,8 \%$ \\
\hline 1 a 2 salários mínimos & 98 & $59,0 \%$ \\
\hline 2 a 3 salários mínimos & 15 & $9,0 \%$ \\
\hline 3 a 4 salários mínimos & 9 & $5,4 \%$ \\
\hline 4 ou mais salários mínimos & 19 & $11,4 \%$ \\
\hline Não respondeu & 12 & $7,2 \%$ \\
\hline
\end{tabular}




\begin{tabular}{lcc} 
Família (incluindo a Idosa) & Média & $\mathrm{R} \$ 2.990,23$ \\
Até 1 salário mínimo & 2 & $1,2 \%$ \\
1 a 2 salários mínimos & 21 & $12,7 \%$ \\
2 a 3 salários mínimos & 30 & $18,1 \%$ \\
3 a 4 salários mínimos & 18 & $10,8 \%$ \\
4 ou mais salários mínimos & 47 & $28,3 \%$ \\
Não respondeu & 48 & $28,9 \%$ \\
Tipo de Renda que a Idosa possui & & \\
Aposentadoria & 123 & $74,1 \%$ \\
Pensão & 43 & $25,9 \%$ \\
Aposentadoria e Pensão & 5 & $3,0 \%$ \\
Trabalho Próprio & 9 & $5,4 \%$ \\
Doações (família, amigos) & 16 & $9,6 \%$ \\
Outras & 25 & $15,0 \%$ \\
Com quem mora & & \\
Sozinho & 17 & $10,2 \%$ \\
Somente com Cônjuge & 12 & $7,2 \%$ \\
Cônjuge e filho(s) & 19 & $11,4 \%$ \\
Cônjuge, filho(s) genro ou nora & 3 & $1,8 \%$ \\
Somente com filho(s) & 18 & $10,8 \%$ \\
Arranjos trigeracionais (idoso, filhos e netos) & 50 & $30,1 \%$ \\
Arranjos intrageracionais (mora & 3 & $1,8 \%$ \\
somente com outros idosos e o cônjuge) & 2 & $1,2 \%$ \\
Somente com os netos (sem filhos) & 2 & $1,2 \%$ \\
Não familiares & 40 & $24,1 \%$ \\
Outros & 25 \\
\hline
\end{tabular}

*Salário mínimo de referência estipulado no ano de 2010 foi de $R \$ 540,00$

\section{Prevalência da fragilidade e associação com fatores sociodemográficos}

Considerando a prevalência de fragilidade, verificou-se que, das 166 idosas participantes do estudo, 65 (39,2\%) não apresentavam fragilidade, $36(21,7 \%)$ eram aparentemente vulneráveis, enquanto $65(39,2 \%)$ apresentavam diferentes níveis de fragilidade, a saber: $39(23,5 \%)$, com fragilidade leve, $13(7,8 \%)$, moderada, e $13(7,8 \%)$, grave.

$\mathrm{Na}$ análise de possível associação entre a fragilidade e a idade, observou-se que os maiores níveis de fragilidade moderada e grave foram verificados entre as idosas mais velhas, ou seja, com 80 anos ou mais, os quais corresponderam, respectivamente, a $53,8 \%$ e $46,2 \%$, verificando-se a correlação estatisticamente significativa entre a idade e a fragilidade $(p$ $<0,001)$.

Tendo em vista a relação entre a fragilidade e os anos de estudo, identificou-se que, entre as 166 idosas, a média e o desvio padrão encontrados para os anos de estudo foram de 9,06 $(6,53)$, para as idosas sem fragilidade, $5,19(5,42)$, para as que estavam aparentemente vulneráveis à fragilidade, 4,16 $(4,94)$, para as que evidenciavam fragilidade leve, $4,89(6,86)$, para as que tinham fragilidade moderada, e $6,43(6,72)$, para aquelas com fragilidade grave. Ao se analisar a correlação entre os escores brutos de fragilidade e a renda, empregando-se o teste Qui-Quadrado, Kruskal-Wallis, constatou-se correlação estatisticamente significativa entre a fragilidade e os anos de estudo $(p=0,001)$, fragilidade e renda do idoso $(p=0,251)$ e da familia $(p=0,001)$.

\section{DISCUSSÃO}

As características das idosas apresentam como achados relevantes o predomínio de mulheres com 80 anos e mais, o baixo nível de escolaridade e de renda, assim como o arranjo familiar multigeracional como cenário de vivência. A população idosa atual teve menores possibilidades de acesso à escola no passado, e suas oportunidades no mercado de trabalho levaram a ocuparem cargos de menor valia, especialmente as mulheres, o que vem determinar menores rendimentos e pior qualidade de vida.

No tocante ao arranjo familiar multigeracional, emerge como um cenário de permutas intergeracionais, característico da população de idosos brasileiros mais pobres, especialmente os paraibanos, em que as idosas com limitações funcionais, pouca autonomia e com problemas crônicos de saúde, em geral, recebem ajuda e, concomitantemente, contribuem para o orçamento familiar com seus benefícios previdenciários. Tal observação é ratificada por pesquisa na qual se verificou que o idoso mais fragilizado perde a independência e a capacidade de morar sozinho ${ }^{(13)}$.

Os resultados revelam, ainda, que a prevalência da fragilidade entre as mulheres apresentou um índice de 39,2\%, que obteve correlação estatisticamente significativa com a idade ( $p<0,001)$. Assim, foram verificados os maiores índices de fragilidade moderada e grave entre as mulheres com 80 anos ou mais, que corresponderam, respectivamente, a $53,8 \%$ e $46,2 \%$. Isso sugere que quanto maior a idade cronológica, maior a tendência à fragilidade.

A despeito disso, alguns estudos ressaltam a escassez de informações sistemáticas referentes à incidência e a prevalência de fragilidade em idosos, principalmente pela falta de consenso de uma definição que possa ser utilizada como screenig em diferentes populações. Desse modo, acrescentam os autores, devido à não homogeneidade dos parâmetros disponíveis para mensurar o fenômeno, as taxas de prevalência existentes correm o risco de não expressarem adequadamente a realidade ${ }^{(6,14)}$.

Quanto aos fatores envolvidos na determinação da fragilidade, a literatura pesquisada evidencia que indivíduos com idade avançada estão mais propensos à velhice fragiliza-

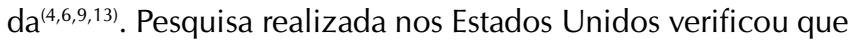
$3 \%$ a $7 \%$ das pessoas com mais de 65 anos de idade eram frágeis. Esse percentual aumenta de $20 \%$ a $26 \%$ em pessoas com mais de 80 anos. Nos idosos com mais de 90 anos, o índice alcança $32 \%{ }^{(5)}$. Isso decorre do fato de os mais velhos, geralmente, apresentarem mais possibilidade de descompensação de sua homeostase quando da ocorrência de eventos agudos, físicos ou psicológicos ${ }^{(15)}$.

Consubstanciando esses achados, o Cardiovascular Health Study, nos Estados Unidos, identificou que, em uma população com idade superior a 65 anos, 6,3\% eram frágeis; entre eles, $68,5 \%$ eram mulheres. Neste estudo, também foi 
observado que a prevalência de fragilidade aumentava com o avançar da idade, de 2,5\% entre 65 e 70 anos para $32 \%$ no grupo com 90 anos ou mais ${ }^{(5)}$.

A maior prevalência de fragilidade em mulheres decorre, especialmente, do fato de sobreviverem mais e manifestarem menor índice de massa muscular, que pode estar relacionado à redução dos níveis de testosterona ${ }^{(16)}$. Além disso, verifica-se que a maior prevalência de fragilidade em idosas é influenciada por condições marcadas por diferenças sexuais, experimentadas por elas ao longo de suas vidas, como o desempenho de atividades domésticas, vida social restrita e pouca independência econômica ${ }^{(7)}$, condições vivenciadas pelas mulheres participantes deste estudo.

A situação ora mencionada é consubstanciada pelos achados deste estudo, porquanto se verificou maior associação da fragilidade com baixo nível de escolaridade ( $p<0,025)$ e renda familiar ( $p<0,001$ ). Diversos estudos descrevem que a incidência de fragilidade em idosos está relacionada ao baixo nível socioeconômico e à pouca escolaridade, por afetar, especialmente, seu estilo de vida e aumentar sua exposição a riscos para a saúde, além de potencializar a condição física da fragilidade ${ }^{(7,18)}$.

Considerando esses aspectos, vários autores ${ }^{(10-11,18-19)}$ reforçam a importância de se considerar a multidimensionalidade do fenômeno, pois sustentam que fatores sociais como renda insuficiente, baixo nível de escolaridade, ausência de apoio social e estilo de vida podem agravar a fisiopatologia da fragilidade. Porém eles asseveram que intervenções apropriadas sobre esses fatores apontam para a possibilidade de se reverter o evento.

Frente ao exposto, em estudos de prevalência deste tipo, podem ocorrer limitações. Desse modo, particularmente no cenário local, é preciso realizar estudos longitudinais para se compreender bem mais os fatores envolvidos na ocorrência do fenômeno da fragilidade, uma vez que o presente estudo apresenta um caráter transversal de observação dos dados, por essa razão, não pode determinar uma rede causal para a fragilidade, constituindo uma limitação.

\section{CONCLUSÃO}

Estudos sobre diferentes aspectos da fragilidade representam uma temática em expansão de interesse especial para os profissionais, particularmente daqueles da área de saúde. A pesquisa em questão possibilitou estimar a prevalência do fenômeno em mulheres idosas, residentes no município de João Pessoa, Paraíba, Brasil e verificar sua associação com idade, escolaridade e renda. Isso contribuiu para se aprofundar a análise de aspectos epidemiológicos e fatores relacionados à fragilidade.

A partir dos achados e das associações verificadas, pode-se sinalizar para o caráter multifatorial do fenômeno investigado, que pode ser compreendido como um desfecho natural do processo fisiológico de envelhecimento ou do declínio da resiliência biológica potencializado por condições sociais qualificadoras daqueles que evidenciam tal problema.

Tais achados podem contribuir efetivamente para o estabelecimento de medidas de prevenção e rastreamento da fragilidade entre as pessoas idosas por parte dos profissionais de saúde, principalmente dos enfermeiros, visando evitar a ocorrência do problema e dos desfechos adversos e indesejáveis resultantes, como a incapacidade, o agravamento das condições de saúde dos idosos, a hospitalização e a institucionalização a ela associados. Essa é, pois, uma forma de se contribuir para um envelhecimento ativo, saudável e com mais qualidade de vida.

\section{REFERÊNCIAS}

1. Silva SLA, Vieira RA, Arantes P, Dias RC. Avaliação de fragilidade, funcionalidade e medo de cair em idosos atendidos em um serviço ambulatorial de geriatria e gerontologia. Fisioter Pesqui. 2009;16(2):125-9.

2. Instituto Brasileiro de Geografia e Estatística [homepage na internet]. Departamento de População e Indicadores Sociais Censo Demográfico 2010. [acesso em jan 2011] Disponível em: http//www.ibge.gov.br

3. Fernandes MGM, Garcia LG. O corpo envelhecido na percepção de homens idosos. Rev Bras Enferm. 2011;64(3):472-7.

4. Gallucci M, Ongaro F, Amici GP, Regini C. Frailty, disability and survival in the elderly over the age of sevently: evidence from the Treviso longeva study. Arch Gerontol Geriatr. 2008;5(106):281-3.

5. Fried L, Tangen CM, Walston J, Newman A, Hirsch C, Mecbrunie MA, et al. Frailty in older adults: evidence for a phenotype. J Gerontol. 2001;56(3):146-56.
6. Macedo M, Gazzola JM, Najas M. Síndrome da fragilidade no idoso: importância da fisioterapia. Arq Bras Ciênc Saúde 2008;33(3):177-84.

7. Woo J, Goggins W, Shan A, Ho SC. Social determinants of frailty. J Gerontol. 2005;51(6):402-8.

8. Hogan D, Macknight C, Bergman H. Models, definitions, and criteria of frailty. Aging 2003;15(3):3-29.

9. Morley JE, Mitchell HP, Miller DK. Something about frailty. J Gerontol. 2002;57(11):698-704.

10. Rockwood K. Frailty and its definition: a worthy challenge. J Am Geriatric Soc 2005;53(6):1067-1070.

11. Rolfson DB, Majumdar SR, Tsuyuki RT, Tahir A, Rockwood K. Validity and reliability of Edmonton frail scale. Age Ageing. 2006;17(5):526-9.

12. Fabrício-Whebe SCC, Schiaveto FV, Vendrúsculo TRP, Haas VJ, Dantas RAS, Rodrigues RAP. Adaptação cultural e validade da Edmonton frail scale - EFS em uma 
amostra de idosos brasileiros. Rev Latino-Am Enferm. 2009;17(6):1043-9.

13. Woods NF, Lacroix AZ, Gray SL, Aragaki A, Cochrane $\mathrm{BB}$, Brunner RL, et al. Frailty: emergence and consequences in women aged 65 and older in the Women's Health Initiative Observational study. J Am Geriatr Soc 2005;53(8):1321-30.

14. Hubbard RE, O'Mahony MS, Woodhouse KW. Characterizing frailty in the clinical setting: a comparison of different approaches. Age Ageing 2009;38(1):115-9.

15. Topinková E. Aging, disability and frailty. Ann Nutr Metab. 2008;52(Suppl 1):6-11.
16. Fernandez-Bolaños $M$, Otero $A$, Zunzunegui $M V$, Beland F, Alarcón T, Hoyos $C$, et al. Sex differences in the prevalence of frailty in a population aged 75 and older in Spain. J Am Geriatric Soc. 2008;36(12):2370-1.

17. Varela-Pinedo L, Ortiz-Saavedra PJ, Chávez-Limeno $\mathrm{H}$. Síndrome de fragilidad em adultos mayores de la comunidad de Lima Metropolitana. Rev Soc Peru Med Interna. 2008;21(1):1-5.

18. Rockwood K. What would make a definition of frailty successful? Age Ageing 2005;34:432-4.

19. Levers MJ, Estabrooks CA, Ross Kerr JC. Factors contributing to frailty: literature review. J Adv Nurs. 2006;56(3):282-91. 